Supplement of Biogeosciences, 17, 2499-2519, 2020

https://doi.org/10.5194/bg-17-2499-2020-supplement

(C) Author(s) 2020. This work is distributed under

the Creative Commons Attribution 4.0 License.

(c) (1)

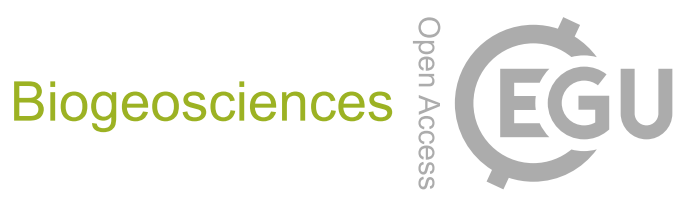

Supplement of

\title{
Patterns of (trace) metals and microorganisms in the Rainbow hydrother- mal vent plume at the Mid-Atlantic Ridge
}

Sabine Haalboom et al.

Correspondence to: Sabine Haalboom (sabine.haalboom@nioz.nl) and David M. Price (d.m.price@ soton.ac.uk)

The copyright of individual parts of the supplement might differ from the CC BY 4.0 License. 


\section{Supplementary material to "Patterns of (trace) metals and microorganisms in the Rainbow hydrothermal vent plume at the Mid-Atlantic Ridge" by Haalboom et al.}

\section{Tables}

Table S1. Dissimilarity (\%) between biotopes based on SIMPER results of microbial classes.

\begin{tabular}{c|cccc} 
& Above Plume & Plume & Below plume & Near-bottom water \\
\hline Above Plume & & & & \\
Plume & 44.83 & & & \\
Below plume & 25.39 & 35.37 & & \\
Near-bottom water & 32.79 & 47.13 & 35.47 & 32.89 \\
Sediment & 44.46 & 56.42 & 46.6 &
\end{tabular}

Table S2-A. Comparison of (trace) metal data with work of German et al. (1991), Edmond et al. (1995) and Edmonds and German (2004).

\begin{tabular}{|c|c|c|c|c|c|c|c|c|c|c|c|c|c|}
\hline Location & Sample & $\begin{array}{l}\text { Depth } \\
{[\mathrm{m}]}\end{array}$ & $\begin{array}{c}\mathbf{F e} \\
{[\mathrm{nM}]}\end{array}$ & $\underset{[n M]}{\mathbf{C a}}$ & $\begin{array}{c}\mathbf{A l} \\
{[\mathrm{nM}]}\end{array}$ & $\begin{array}{c}\text { Mn } \\
{[\mathrm{pM}]}\end{array}$ & $\begin{array}{c}\mathbf{V} \\
{[\mathrm{pM}]}\end{array}$ & $\underset{[p M]}{\mathbf{C u}}$ & $\underset{[p M]}{\mathbf{Z n}}$ & $\begin{array}{c}\text { Co } \\
{[\mathrm{pM}]}\end{array}$ & $\begin{array}{c}\mathbf{P b} \\
{[\mathrm{pM}]}\end{array}$ & $\begin{array}{c}\mathbf{Y} \\
{[\mathbf{p M}]}\end{array}$ & Reference \\
\hline TAG & 14 & 3477 & 56 & 34 & 1.4 & 140 & 260 & 980 & & 9 & 15 & 3.5 & $\begin{array}{l}\text { German et al. } \\
\text { (1991) }\end{array}$ \\
\hline TAG & 18 & 3364 & 87 & 39 & 1.2 & 140 & 393 & 620 & 205 & 8 & & 6.7 & $\begin{array}{c}\text { German et al. } \\
\text { (1991) }\end{array}$ \\
\hline TAG & 19 & 3392 & 67 & 35 & 1.4 & & 323 & 760 & 167 & 6 & 11 & 3.7 & $\begin{array}{l}\text { German et al. } \\
\text { (1991) }\end{array}$ \\
\hline TAG & 22 & 3337 & 192 & 53 & 1.6 & 180 & 888 & 15440 & 512 & 71 & 21 & 8.7 & $\begin{array}{c}\text { German et al. } \\
\text { (1991) }\end{array}$ \\
\hline TAG & $403 \mathrm{~T}$ & 3340 & 50 & & 0.52 & 189 & 239 & 1405 & & & & & $\begin{array}{c}\text { Edmond et al. } \\
(1995)\end{array}$ \\
\hline TAG & 403B & 3440 & 38 & & 0.62 & 193 & 174 & 647 & & & & & $\begin{array}{c}\text { Edmond et al. } \\
(1995)\end{array}$ \\
\hline TAG & $409 \mathrm{~T}$ & 3081 & 4 & & 1.06 & 190 & 32 & 40 & & & & & $\begin{array}{c}\text { Edmond et al. } \\
\text { (1995) }\end{array}$ \\
\hline TAG & 409B & 3231 & 5 & & 0.3 & 339 & 27 & 20 & & & & & $\begin{array}{c}\text { Edmond et al. } \\
\text { (1995) }\end{array}$ \\
\hline Rainbow & $\begin{array}{l}\text { SAP } \\
05 \_1\end{array}$ & 2025 & 278.8 & 83.6 & 0.3 & 184 & 1389 & 2386 & 287 & 47.2 & 24.5 & 13 & $\begin{array}{c}\text { Edmonds and } \\
\text { German (2004) }\end{array}$ \\
\hline Rainbow & $\begin{array}{l}\text { SAP } \\
06 \_1\end{array}$ & 1940 & 26.4 & 51 & 1 & 144 & 143 & 134 & 178 & 4.1 & 19.4 & 2.3 & $\begin{array}{l}\text { Edmonds and } \\
\text { German (2004) }\end{array}$ \\
\hline Rainbow & $\begin{array}{l}\text { SAP } \\
07 \_1\end{array}$ & 2150 & 18 & 72.2 & 3.4 & 216 & 98 & 153 & & 5 & 24.6 & 2.4 & $\begin{array}{l}\text { Edmonds and } \\
\text { German (2004) }\end{array}$ \\
\hline Rainbow & $\begin{array}{l}\text { SAP } \\
09 \_1\end{array}$ & 2100 & 128.4 & 38.6 & 0.9 & 45 & 504 & 1781 & 751 & 43.5 & 7.2 & 4.1 & $\begin{array}{l}\text { Edmonds and } \\
\text { German (2004) }\end{array}$ \\
\hline Rainbow & 27 & 2077 & 355.43 & 700.31 & 2.15 & 202.78 & 1910.64 & 5355.68 & 2030.09 & 117.40 & 32.97 & 15.61 & This study \\
\hline Rainbow & 42 & 2209 & 38.42 & 446.55 & 0.04 & 22.47 & 205.65 & 396.33 & 25.47 & 15.71 & & 0.97 & This study \\
\hline Rainbow & 44 & 2002 & 132.73 & 1605.10 & 2.14 & 263.64 & 894.23 & 1355.13 & 729.65 & 77.71 & 37.23 & 10.05 & This study \\
\hline Rainbow & 45 & 2166 & 171.11 & 1052.82 & 1.19 & 116.28 & 1213.40 & 1487.52 & 81.95 & 44.95 & 28.69 & 12.81 & This study \\
\hline Rainbow & 46 & 2280 & 139.98 & 455.14 & 1.67 & 129.49 & 917.24 & 1195.15 & 353.27 & 31.14 & 26.29 & 9.99 & This study \\
\hline
\end{tabular}


Table S2-B. Comparison of REE data with work of Edmonds and German (2004).

\begin{tabular}{|c|c|c|c|c|c|c|c|c|c|c|c|c|c|c|c|c|c|}
\hline Location & Sample & $\begin{array}{c}\text { Depth } \\
{[\mathrm{m}]}\end{array}$ & $\begin{array}{c}\mathbf{L a} \\
{[\mathbf{p M}]}\end{array}$ & $\begin{array}{c}\mathrm{Ce} \\
{[\mathrm{pM}]}\end{array}$ & $\begin{array}{c}\mathrm{Pr} \\
{[\mathrm{pM}]}\end{array}$ & $\begin{array}{c}\text { Nd } \\
{[\mathbf{p M}]}\end{array}$ & $\begin{array}{c}\text { Sm } \\
{[\mathbf{p M}]}\end{array}$ & $\begin{array}{c}\mathbf{E u} \\
{[\mathbf{p M}]}\end{array}$ & $\begin{array}{c}\text { Gd } \\
{[\mathbf{p M}]}\end{array}$ & $\begin{array}{c}\text { Tb } \\
{[\mathbf{p M}]}\end{array}$ & $\begin{array}{c}\text { Dy } \\
{[\mathbf{p M}]}\end{array}$ & $\begin{array}{c}\text { Ho } \\
{[\mathbf{p M}]}\end{array}$ & $\begin{array}{c}\mathbf{E r} \\
{[\mathbf{p M}]}\end{array}$ & $\begin{array}{c}\mathbf{T m} \\
{[\mathbf{p M}]}\end{array}$ & $\begin{array}{c}\mathbf{Y b} \\
{[\mathbf{p M}]}\end{array}$ & $\begin{array}{c}\mathbf{L u} \\
{[\mathbf{p M}]}\end{array}$ & Reference \\
\hline Rainbow & SAP05_1 & 2025 & 6.830 & 3.630 & 1.330 & 5.190 & 0.951 & 0.379 & 0.823 & 0.150 & 0.917 & 0.196 & 0.543 & 0.072 & 0.418 & 0.061 & $\begin{array}{c}\text { Edmonds } \\
\text { and } \\
\text { German } \\
(2004)\end{array}$ \\
\hline Rainbow & SAP06_1 & 1940 & 1.180 & 1.290 & 0.272 & 1.117 & 0.217 & 0.071 & 0.203 & 0.035 & 0.194 & 0.038 & 0.108 & 0.013 & 0.093 & 0.013 & $\begin{array}{l}\text { Edmonds } \\
\text { and } \\
\text { German } \\
(2004)\end{array}$ \\
\hline Rainbow & SAP07_1 & 2150 & 1.540 & 2.380 & 0.392 & 1.563 & 0.293 & 0.083 & 0.225 & 0.041 & 0.229 & 0.044 & 0.121 & 0.015 & 0.091 & 0.012 & $\begin{array}{l}\text { Edmonds } \\
\text { and } \\
\text { German } \\
(2004)\end{array}$ \\
\hline Rainbow & SAP09_1 & 2100 & 2.300 & 1.380 & 0.439 & 1.788 & 0.330 & 0.180 & 0.294 & 0.050 & 0.307 & 0.064 & 0.174 & 0.022 & 0.137 & 0.018 & $\begin{array}{l}\text { Edmonds } \\
\text { and } \\
\text { German } \\
(2004)\end{array}$ \\
\hline Rainbow & 27 & 2077 & 7.179 & 4.343 & 1.389 & 5.250 & 1.019 & 0.498 & 1.149 & 0.193 & 1.285 & 0.274 & 0.717 & 0.093 & 0.521 & 0.072 & This study \\
\hline Rainbow & 42 & 2209 & 0.480 & & 0.124 & & 0.090 & 0.036 & 0.102 & 0.010 & 0.077 & 0.024 & 0.054 & 0.006 & 0.043 & 0.003 & This study \\
\hline Rainbow & 44 & 2002 & 5.562 & 3.247 & 1.160 & 4.037 & 0.842 & 0.302 & 0.984 & 0.147 & 0.956 & 0.228 & 0.554 & 0.068 & 0.410 & 0.051 & This study \\
\hline Rainbow & 45 & 2166 & 6.130 & 3.305 & 1.308 & 4.658 & 0.979 & 0.375 & 1.148 & 0.187 & 1.252 & 0.271 & 0.694 & 0.089 & 0.526 & 0.072 & This study \\
\hline Rainbow & 46 & 2280 & 4.884 & 2.972 & 1.059 & 3.839 & 0.803 & 0.303 & 0.933 & 0.155 & 0.976 & 0.205 & 0.537 & 0.070 & 0.415 & 0.059 & This study \\
\hline
\end{tabular}




\section{Figures}

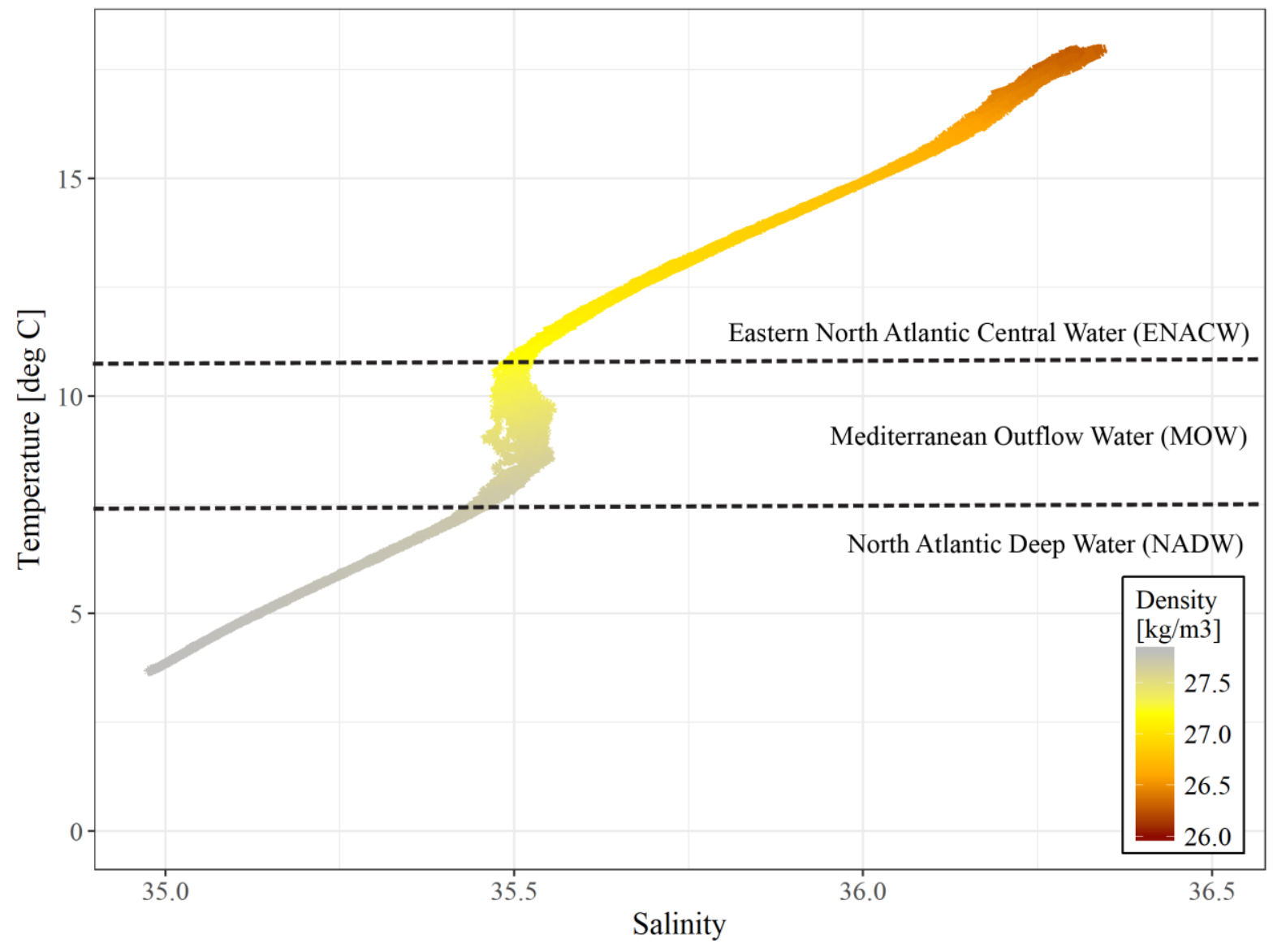

Figure S1: Temperature - salinity plot of all CTD casts showing the three distinct water masses found in the Rainbow area. 

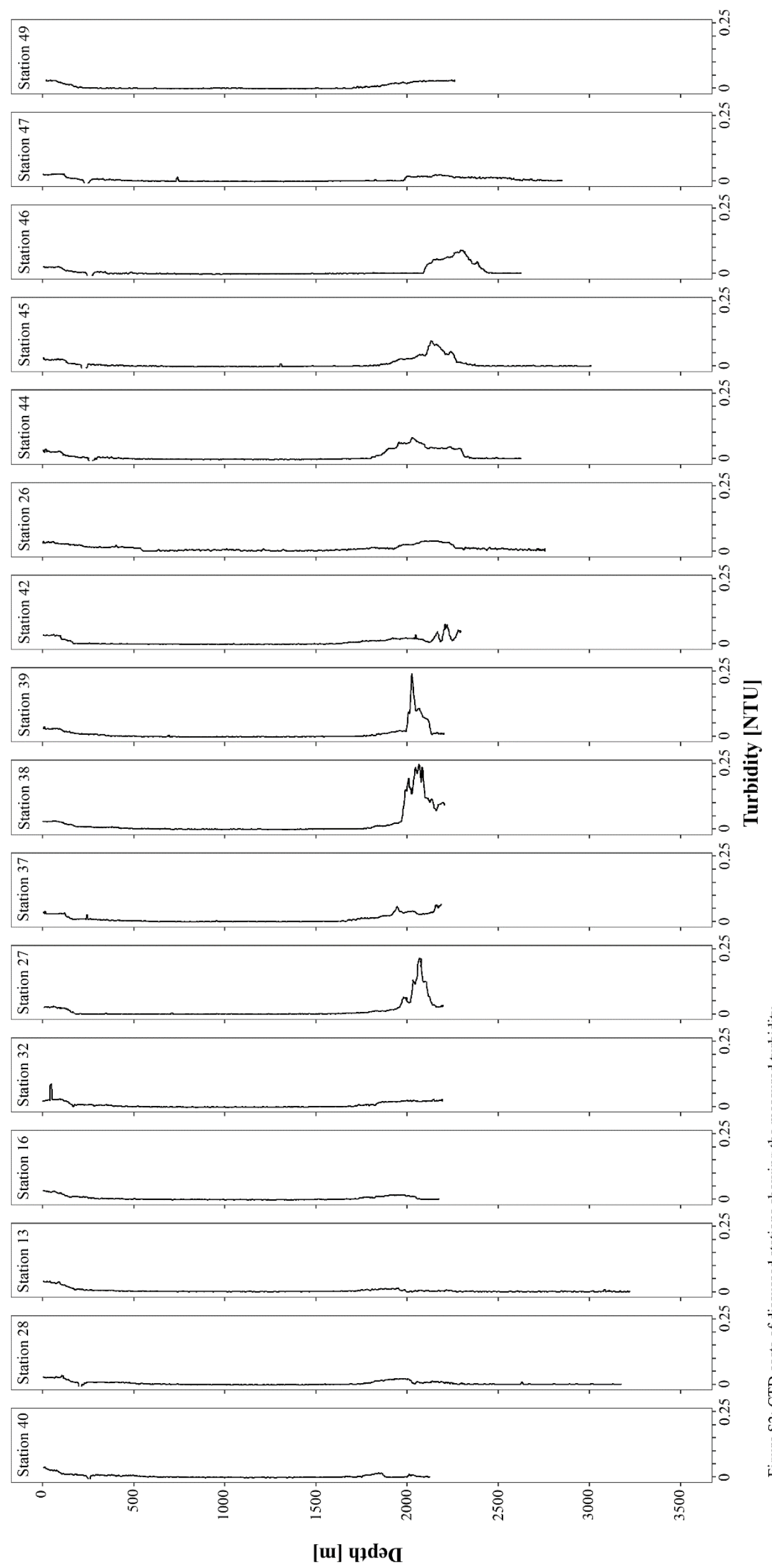

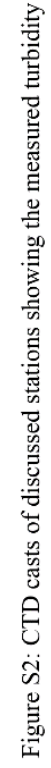

[u] чłdәd 

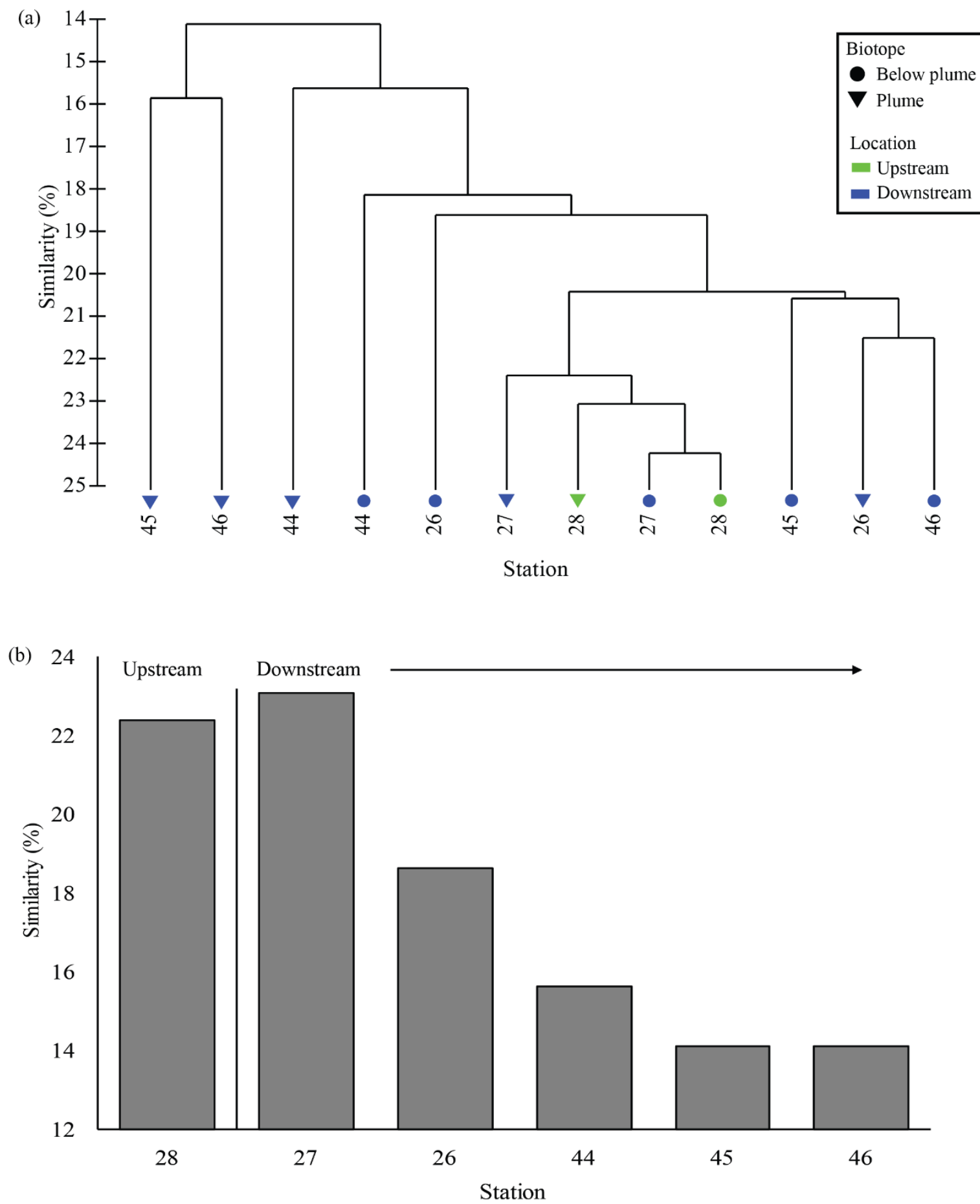

Figure S3: Group average clustering of plume and below plume microbial communities. (a) Dendrogram results (b) Similarity between plume and below plume samples. 


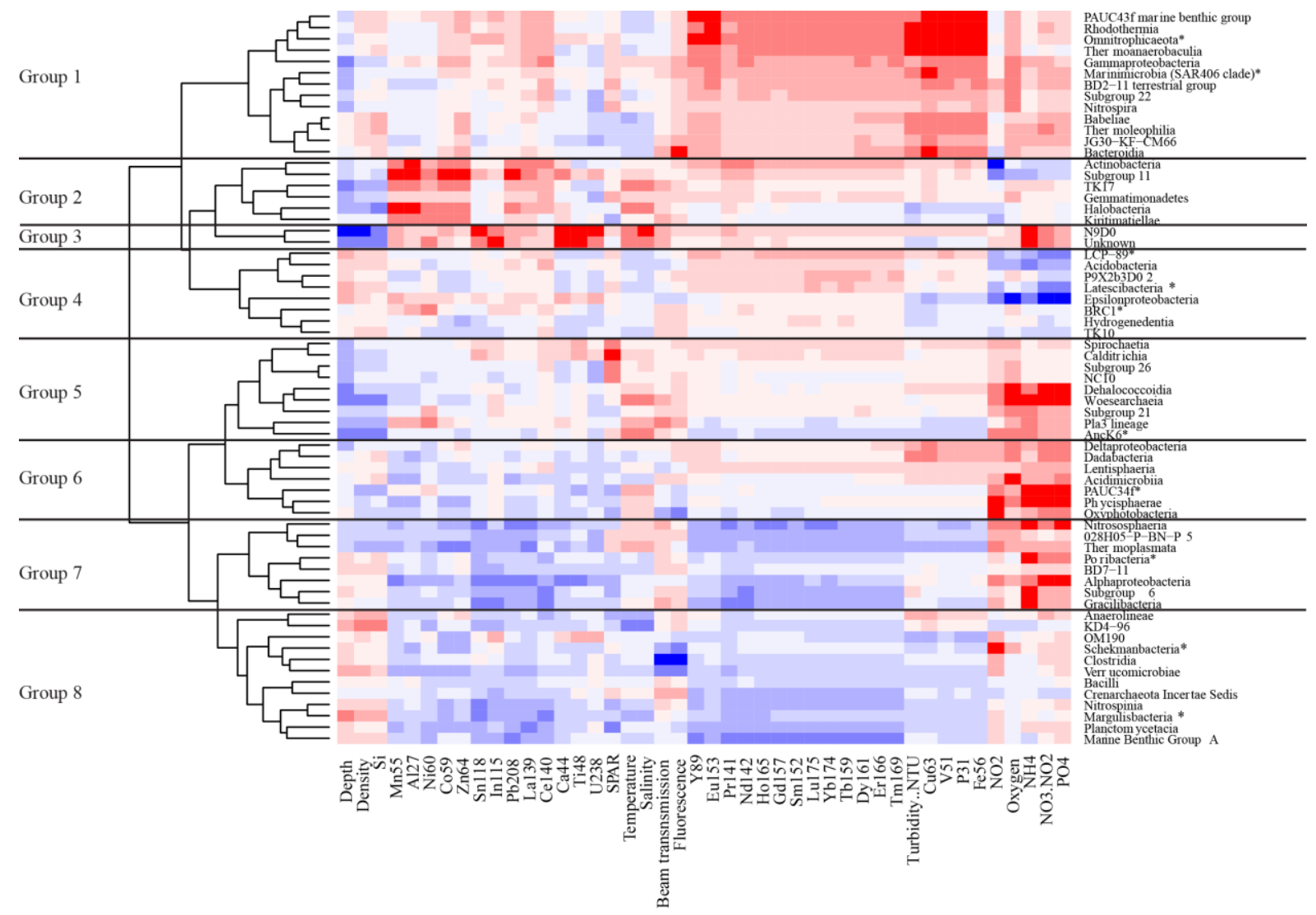

Figure S4: Pearson correlation heatmap of the correlation between all environmental variables and all classes that were present in multiple samples within plume samples. Colour intensity refers to the strength of the correlation, red indicates a positive correlation and blue indicates a negative correlation. Dendrograms based on hierarchical clustering and groups based on clustering to 8 distinct groups. 